\title{
MODELOS MATEMÁTICOS APLICADOS NO CRESCIMENTO DE COLMÉIAS DE APIS MELLIFERA L. NO CARIRI PARAIBANO
}

\author{
APPLIED MATHEMATICAL MODELS IN THE GROWTH OF BEEHIVES OF \\ APIS MELLIFERA L. IN CARIRI PARAIBANO
}

\author{
Souza, D.N. ${ }^{1 A}$, Evangelista-Rodrigues, A. ${ }^{1 *}$, Cruz, G.R.B. ${ }^{1}$, Rodrigues, M.L. ${ }^{1}$, \\ Silva, M.C. ${ }^{1}$ e Gois, G.C. ${ }^{1}$
}

'Departamento de Zootecnia. Centro de Ciências Agrárias. Universidade Federal da Paraíba. CEP 58937000. Areia, PB. Brasil. *adriana@cca.ufpb.br; Adenizenubia@gmail.com

\section{Palavras chave adicionals}

Abelha. Manejo de colméias. Modelagem de crescimento.

\section{RESUMO}

Buscando avaliar a potencialidade da apicultura no Cariri Paraibano, objetivou-se a utilização de modelos matemáticos adequados para estimar o crescimento das colônias. Os dados foram tomados semanalmente com a pesagem de ninhos de 20 colméias instaladas na Estação Experimental de São João do Cariri (CCA/UFPB). A estimativa dos parâmetros foi feita por meio de regressões não-lineares, pelo procedimento NLIN do programa SAS (Statistical Analysis System), utilizandose o método modificado de Gauss-Newton. Para a escolha do melhor modelo, utilizou-se número de interações, o coeficiente de determinação $\left(R_{a}^{2}\right)$ e o gráfico de distribuição de resíduo. Entre os modelos estudados, o que apresentou melhor ajuste foi a quadrático logarítmico.

\section{SUMMARY}

The aim of this work was to evaluate the potentiality of the beekeeping in the Cariri Paraibano, using mathematical models to esteem the growth of the colonies. The data had been taken weekly with the weighing of nests of 20 beehives installed in the Experimental Station (CCA/UFPB) of São João do Cariri. The estimate of the parameters was made by means of nonlinear regressions, for procedure NLIN of Program SAS (Statistical Analysis System), using the modified GaussNewton method. For the choice of the best model, the number of interactions, the determination coefficient and the residue distribution graph were

Recibido: 8-7-08. Aceptado: 22-10-08.

\section{AdDitionAL KEYWORDS}

Bee. Handling of beehives. Growth modelling.

used. Among the studied models, better adjustment was achieved with the quadratic logarithmic model.

\section{INTRODUÇÃO}

A apicultura é uma atividade dependente dos recursos naturais, possuindo oscilações de produção de acordo com as condições climáticas e ambientais de cada região. Assim, para que essa atividade possa ser expressiva quanto à produção é necessário que o produtor tenha conhecimento acerca da disponibilidade de recurso (néctar e pólen) fornecido pela vegetação e qual a real participação destas na produção anual da região.

O conhecimento da produção apícola, por seu turno, permite que o manejo das colméias seja conduzido eficientemente, possibilitando, assim, que programas de seleção de abelhas sejam elaborados para as características de crescimento e produção das colméias.

A utilização de modelos matemáticos em colméias de Apis mellifera pode contribuir para o melhor entendimento do sistema de produção, uma vez que o conhecimento da forma da curva e suas implicações sobre o crescimento do ninho podem auxiliar na estimativa de crescimento máximo em deter- 
minado período de tempo e, também, na tomada de decisões quanto ao manejo, haja visto que o estudo de curvas de crescimento por meio de modelos matemáticos, geralmente, sintetiza informações de todo o período de vida dos animais, ou seja, fornece um conjunto de informações em série de peso e tempo, cujo conjunto de parâmetros são interpretáveis biologicamente, facilitando o entendimento do fenômeno (Oliveira et al., 2000; Cobuci et al., 2001).

Assim posto, busca-se neste trabalho aplicar modelos matemáticos que melhor estimem o crescimento dos ninhos de $A$. mellifera, visando contribuir para o direcionamento do manejo adequado para a região do Cariri Paraibano.

\section{MATERIALEMÉTODOS}

O experimento foi conduzido no apiário da Estação Experimental de São João do Cariri, pertencente ao Centro de Ciências Agrárias (CCA) da Universidade Federal da Paraíba(UFPB), durante o período de 12 de abril a 6 de setembro de 2007.

Durante o experimento foram utilizadas 20 colméias nidificadas em caixas modelo Langstroth, onde estas receberam manejo contínuo de produção sem a intervenção de novas famílias. As colônias eram naturais, capturadas na região, não sofreram substituição de rainhas e a fonte de alimentação foi a flora local. Não houve necessidade de nenhuma medicação ao longo do período experimental.

Para estimar o crescimento das colônias, semanalmente no período vespertino, foram realizadas as pesagens dos ninhos diretamente no campo, utilizando-se balança eletrônica com bateria para carga máxima de $30 \mathrm{~kg}$, totalizando 22 semanas.

Sete funções matemáticas foram utilizadas para ajustar as pesagens (gama incompleta, logística, linear, linear hiperbólica, quadrática, quadrática logarítmica e polinomial inversa). Os dados médios das pesagens semanais das unidades experimentais foram tabulados em planilhas no programa Excel para posterior avaliação estatística.

Os parâmetros das funções foram estimados através de técnicas não-lineares, usando-se o método modificado de GaussNewton disponível no PROC NLIN (SAS, 1999-2001). A escolha do melhor modelo foi determinada pelo coeficiente de determinação ajustado, número de interações, gráfico de distribuição dos resíduos e desvios entre as pesagens observadas e estimadas, os quais contribuíram para a tomada de decisões quanto à qualidade de ajuste proporcionada por uma função.

\section{RESULTADOSEDISCUSSÃO}

A média de peso dos ninhos de Apis mellifera foi $6,169 \mathrm{~kg}$, em um período de 22 semanas, com coeficiente de variação de $38,56 \%$. A curva de crescimento do ninho está representada na figura 1 , onde se percebe flutuações no crescimento ao longo do tempo, que frequentemente resultam de mudanças sazonais ou anuais na disponibilidade de recursos, ou podem ser aleatórias. As populações de insetos não são constantes; eles apresentam períodos de crescimento quando o ambiente lhes é favorável e decréscimos, quando sobrepujam os fatores desfavoráveis. Portanto, num determinado espaço de tempo as populações flutuam, na dependência dos fatores relativos a clima, principalmente temperatura (Azerefegne et al., 2001). No entanto, diversos estudos de modelagem têm revelado a ocorrência de interações ecológicas, dinâmicas intrínsecas ou mesmo outros fatores não climáticos, regulando as oscilações de indivíduos (Turchin et al., 1991).

As médias dos parâmetros estudados nos diversos modelos permitem a interpretação biológica dos dados para ressaltar características relevantes para o estudo do crescimento ou produção (Tedeschi et al., 2000). Assim, $a_{0}$ corresponde ao peso inicial estimado pelo modelo o qual foi aproximado 


\section{MODELOS MATEMÁTICOS PARA CRESCIMENTO DE APIS MELLIFERA}
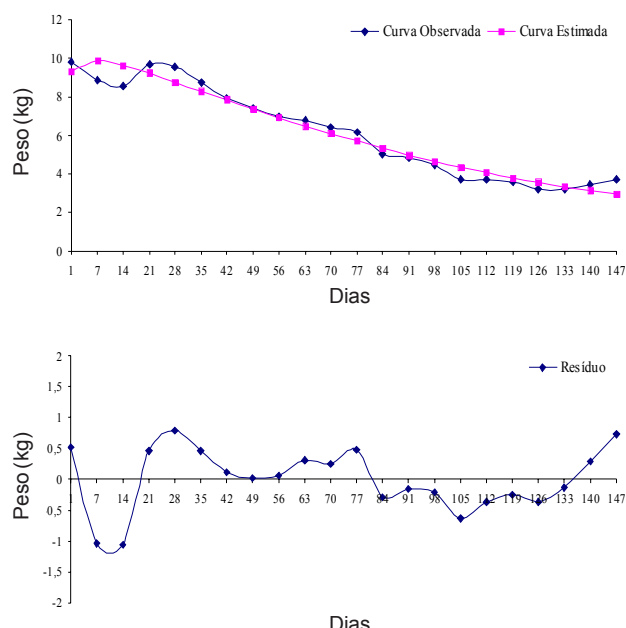

Figura 1. Curvas de crescimento estimado e observado, e média do resíduo para o modelo quadrático logarítmico. (Observed and estimated growth curve, and mean of residual for the model quadratic logarithmic).

ao peso inicial observado nos modelos linear e o linear hiperbólico $(9,901$ e 9,926 kg, respectivamente).

Quanto ao número de interações, este varou de 2 a 15 , sendo os menores valores atribuídos para os modelos linear, linear hiperbólico, quadrático e quadrático logarítmico com duas interações cada. Esses valores demonstram o grau de dificuldade computacional no ajuste dos dados.

Dentre os modelos estudados, houve variação do coeficiente de variação ajustado $\left(R_{a}^{2}\right)$ de 78,3 a $80,2 \%$, sendo o quadrático logarítmico aquela que apresentou maior valor $(80,2 \%)$, seguido do linear $(79,3 \%)$. De acordo com Cruz (2007), o $R_{a}^{2}$ indica o quanto da variação total foi explicada pelo modelo de regressão e elevados valores de $R_{a}^{2}$ indicam bons ajustes.

A análise do coeficiente de determinação ajustado $\left(R_{a}^{2}\right)$, o número de interações, a avaliação gráfica das curvas estimada e observada e do gráfico do resíduo mostram bom ajuste do modelo quadrático logarítmico. A figura 1 apresenta as curvas de crescimento médio observado e estimado e o resíduo do modelo quadrático logarítmico, onde nota-se uma boa representação de sua estrutura quando comparada aos demais modelos. Os dados demonstram subestimação do peso inicial e final em 0,510 e 0,73 $\mathrm{kg}$, respectivamente; já com relação ao resíduo, este variou de $-1,065$ a $0,784 \mathrm{~kg}$.

$O$ formato da curva demonstra as flutuações ocorridas ao longo do tempo, sendo esta caracterizada por acentuado decréscimo sofrido a partir da quinta semana e que se prolonga até a décima nona semana. Esse decréscimo da curva é decorrente do período de estiagem da região e como conseqüência a escassez de vegetação em florescimento. De acordo com Silveira-Neto et al. (1976), a fase de decréscimo de crescimento em insetos ocorre quando o aumento populacional é lento e se aproxima de uma assintota. É nesta fase que pode ocorrer flutuações, que são afastamentos assimétricos do nível de equilíbrio.

A partir da vigésima semana de observação (figura 1) é percebido leve acréscimo do peso das colméias estudadas, decorrente do florescimento da espécie vegetal exótica Prosopis juliflora (algaroba), que teve início no o mês de agosto durante o período de estiagem na região.

Segundo Free (1980), como resultado do aumento do número de crias que é acompanhada pelo aumento de coleta de recursos, a população adulta de abelhas aumenta, e se a coleta permanecer abundante, assim também o serão o número de abelhas coletoras e a quantidade de alimento coletado. Por sua vez, a quantidade de alimento coletado é influenciada pela quantidade de crias presentes na colméia.

À medida que recursos florais (pólen e néctar) vão sendo disponibilizados para a colônia, estas tendem a aumentar o número de indivíduo, sendo seu o crescimento relacionado à oferta de alimentos. O declínio da população é decorrente da escassez de recursos ofertados a colméia. 


\section{SOUZA, EVANGELISTA-RODRIGUES, CRUZ, RODRIGUES, SILVAE GOIS}

A importância do estudo de curvas de crescimento em abelhas $A$. mellifera reside em permitir a utilização de manejos adequados com base na variação do crescimento das colméias em função do tempo e do ambiente, possibilitando a determinação de meses do ano cujos valores máximos e mínimos de crescimento serão determinados. Com esses resultados, é possível traçar estratégias de manejos que viabilizem a produção, haja visto que em colméias com número elevado de abelhas operárias e crias a produção é maior se comparada à uma colméia com menor número de indivíduos. Outra informação a ser considerada através deste estudo é a determinação dos períodos de entressafra ( $5^{\mathrm{a}}$ a $19^{\mathrm{a}}$ semana $)$ e safra $\left(1^{\mathrm{a}}\right.$ a $4^{\mathrm{a}}$ semana e a partir da $20^{\mathrm{a}}$ semana). Relacio-

\section{BIBLIOGRAFIA}

Azerefegne, F., Solbreck, C. and Ives, A.R. 2001. Environmental forcing and high amplitude fluctuations in the population dynamics of the tropical butterfly Acraea acerata (Lepidoptera: Nymphalidae). J. Anim. Ecol., 70: 1032-1045

Cobuci, J.A., Euclydes, R.F., Teodoro, R.L., Verneque, R.S., Lopes, P.S. e Silva, M.A. 2001. Aspectos genéticos e ambientais da curva de lactação de vacas da raça Guzerá. Rev. Bras. Zootecn., 30: 1204-1211.

Cruz, G.R.B. 2007. Estimativas dos parâmetros genéticos e de ambiente para medidas repetidas de produção de leite e de gordura em bovinos da raça Sindi e Guzerá. (Tese de Doutorado). Universidade Federal Rural de Pernambuco. Recife. 135 pp.

Free, B.J. 1980. A organização social das abelhas (Apis). Editora da Universidade de São Paulo, São Paulo. 13: 79. nando essas informações com a oferta de recursos e condições climáticas, percebese que a partir da quinta semana houve menor período de chuva, ocasionando um menor número de floração com conseqüente diminuição na oferta de néctar e pólen. Enquanto que nas quatro primeiras semanas e na vigésima semana ocorreu o contrário, sendo caracterizada por leves flutuações do crescimento.

\section{CONCLUSÕES}

Dentre os modelos estudados, podemos concluir que o quadrático logarítmico foi o modelo que melhor descreveu o comportamento da curva de crescimento para colméias de $A$. mellifera nas condições estudadas.

Oliveira, H.N.L., Lôbo, R.B. e Pereira, C.S. 2000. Comparação de modelos não lineares para descrever o crescimento de fêmeas da raça Guzerá. Pesqui. Agropecu. Bras., 35: 18431851.

Silveira-Neto, S., Nakano, O., Barbin, D. e Nova, N.A.V. 1976. Manual de ecologia dos insetos. Agronômica Ceres. São Paulo. 419 pp.

SAS. 1999-2001.Statistical Analyses System User's guide: statistics. Version 8, v. 2. Cary.

Tedeschi, L.O., Boin, C., Nadon, R.F. e Leme, P.R. 2000. Estudo da curva de crescimento de animais da raça Guzerá e seus cruzamentos alimentados a pasto, com e sem suplementação. 1 Análise e seleção das funções não-lineares. Rev. Bras. Zootecn., 29: 630-637.

Turchin, P., Lorio Junior, P.L., Taylor, A.D. e Billings, R.F. 1991. Why do populations of southern pine beetles (Coleóptera: Scolytidae) fluctuate? Environ. Entomol., 20: 401-409. 